\title{
ESTIMATION OF PARAMETERS IN COMPOUND WEIBULL DISTRIBUTIONS
}

By Lee W. Falls

Aero-Astrodynamics Laboratory

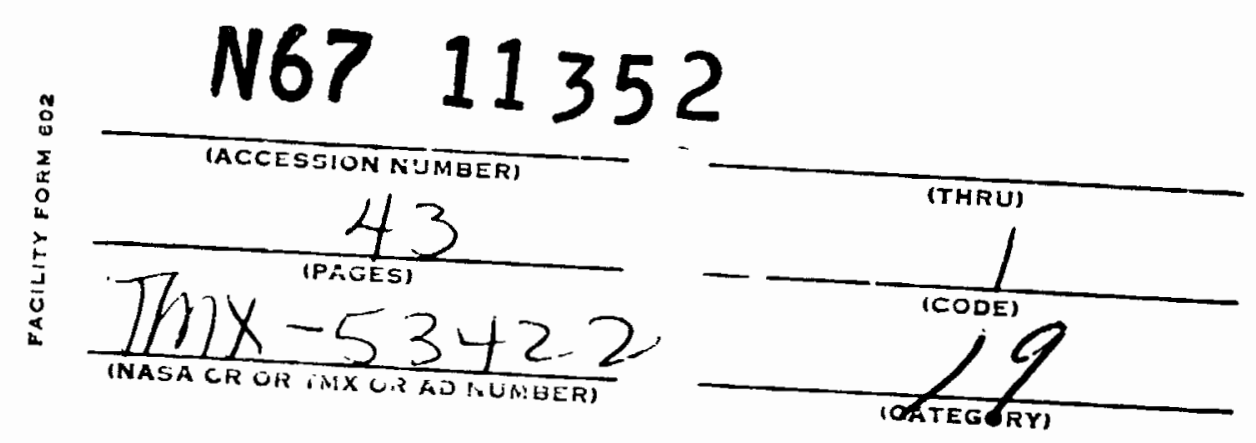

NASA

George C. Marshall GPO PRICE \$

Space Flight Center, Huntsville, Alabama CFSTI PRICE(S) $\$$

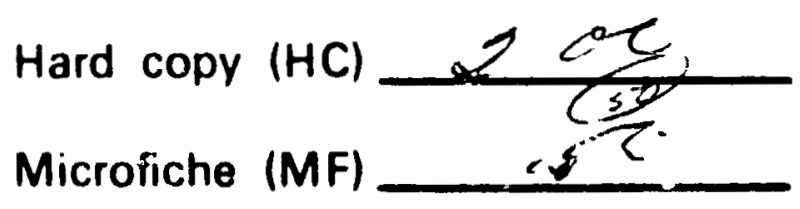


TECHNICAL MEMORANDUM X-53422

ESTIMATION OF PARAMETERS IN COMPOUND WEIBULL DISTRIBUTIONS

By

Lee W. Falls

George C. Marshall Space Flight Center

Huntsville, Alabama

\begin{abstract}
The two-parameter Weibull distribution has been recognized as a useful model for survival populations associated with reliability studies and life testing experiments. In the analys is of atmospheric data, the distributions encouniered are often a result of crmbining two or more component distributions. These compound distribucions aie consequently of interest to aerospace scientists. Presented is a method for estimation of the parameters of a compound Weibull distribution with two shape parameters, two scale parameters and a proportionality factor. The most general case of estimation will be considered in addition to a number of special cases that may be of practical value.
\end{abstract}




\begin{abstract}
NASA - GEORGE C. MARSHALL SPACE FLIGHT CENTER
\end{abstract}
TECHNICAL MEMORANDUM X-53422

March 30, 1966

ESTIMATION OF PARAMETERS IN COMPOUND WEIBULL DISTRIBUTIONS

By

Lee W. Fal1s 
TABLE OF CONTENTS

Page

SUMMARY

I. INTRODUCTION 2

II. ESTIMATION IN THE GENERAL CASE 3

III. ESTIMATION IN SPECIAL CASES 9

IV. AN ILLUSTRATIVE EXAMPLE 19

$\begin{array}{ll}\text { V. CONCLUSIONS } & 26\end{array}$

$\begin{array}{ll}\text { APPENDIX } & 27\end{array}$

$\begin{array}{ll}\text { REFERENCES } & 33\end{array}$ 
TECHNICAL MEMORANDUM X-53422

ESTIMATION OF PARAMETERS IN COMPOUND WEIBULL DISTRIBUTIONS

SUMMARY

The two-parameter Weibull distribution has been recognized as a useful model for survival populations associated with reliability studies and life testing experiments. In the analysis of atmospheric data, the distributions encountered are often a result of combining two or more component distributions. These compound distributions are consequently of interest to aerospace scientists. This paper presents a method for estimation of the parameters of a compound Weibull distribution with density function

$$
f(x)=\alpha f_{I}(x)+(1-\alpha) f_{2}(x), \quad 0<\alpha<1
$$

where

$$
f_{1}(x)=\gamma_{1} \theta_{1}^{-1} x^{\gamma_{1}-1} \exp \left[-x^{\gamma_{1} / \theta_{1}}\right], \quad x \geq 0, \theta_{1}>0, \gamma_{1}>0
$$

and

$$
f_{2}(x)=\gamma_{2} \theta_{2}^{-1} x^{\gamma_{2}-1} \exp \left[-x^{\gamma_{2}} / \theta_{2}\right], \quad x \geqq 0, \theta_{2}>0, \gamma_{2}>0
$$

The parameters required are $\alpha$, the proportionality factor, $\gamma_{1}, \gamma_{2}, \theta_{1}$ and $\theta_{2}$. The most general case of estimation will be considered in addition to a number of special cases that may be of practical value. 


\section{INTRODUCTION}

The Weibull distribution, derived in 1939 by $W$. Weibull, has been recognized as an appropriate model in reliability studies and life testing. Numerous methods for obtaining efficient estimates of the two parameters of this distribution have been outlined in recent years [4, $10,11]$.

In actual physical applications, however, a mixture of two Weibull distributions often seems to be a more desirable model. Distributions resulting from mixing two or more component distributions are designated as "mixed" or "compound." This situation is quite common in the analys is of atmospheric data and consequently is of interest to aerospace scientists. Compound normal, Poisson and exponential distributions have been studied by A. C. Cohen, Jr. $[1,2,3]$. A method for estimating parameters of mixed distributions using sample moments has been outlined by Paul R. Rider [7] who considered compound Poisson, binomial, and a special case of the compound Weibull distribution. A graphical procedure for estimation of mixed Weibull parameters in life-testing of electron tubes is given by John H. K. Kao [6]. Although graphical methods have value for locating outliers, deriving initial estimates, and for determining whether the distribution is as hypothesized, for estimation purposes the analytic approach is probably superior.

This paper represents an attempt at estimating, by the method of sample moments, the five parameters of the compound Weibull distribution with density function

$$
f(x)=\alpha f_{1}(x)+(1-\alpha) f_{2}(x), \quad 0<\alpha<1
$$

where

$$
\left.\begin{array}{l}
f_{1}(x)=\gamma_{1} \theta_{1}^{-1} x^{\gamma_{1}^{-1}} \exp \left[-x^{\gamma_{1} / \theta_{1}}\right], \quad x \geqq 0, \theta_{1}>0, \gamma_{1}>0 \\
f_{2}(x)=\gamma_{2} \theta_{2}^{-1} x^{\gamma_{P^{-1}}} \exp \left[-x^{\gamma_{2} / \theta_{2}}\right], \quad x \geqq 0, \theta_{2}>0, \gamma_{2}>0
\end{array}\right\} .
$$

The parameters involved are two scale parameters $\theta_{1}$ and $\theta_{2}$, two shape parameters $\gamma_{1}$ and $\gamma_{2}$, and the proportionality parameter $\alpha$ which expresses the probability that a given observation $x_{i}$ comes from the population $f_{1}$. 
The compound cumulative distribution function is defined

$F(x)=\alpha F_{1}(x)+(1-\alpha) F_{2}(x)=1-\alpha \exp \left[-x^{\gamma / \theta_{1}}\right]-(1-\alpha) \exp \left[-x^{\gamma / 0_{2}}\right]$

Figures 1 and 2 illustrate a generalized mixed Weibull probability density function and its colresponding distribution function.

The most general case of estimation will be considered in which all five parameters must be estimated from the data. Also, a number of special cases will be investigated in which certain parameters are know in advance of sampling or are restricted in some manner. Included will be the spectal case where $\gamma_{1}=1$, i.e.,

$$
f_{1}(x)=\theta_{1}^{-1} \exp \left[-x / \theta_{1}\right]
$$

which is the well-known exponential distribution. Estimating procedures are greatly simplified in these special cases as there are fewer sample moments involved in the estimating equations. Also, sampling errors are reduced because of the elimination of the need for higher order moments.

\section{ESTTMATION IN THE GENERAL CASE}

The rth theoretical moment about the origin of $f(x)$ is given by

$$
\mu_{r}^{\prime}=\alpha \int_{0}^{\infty} x^{r} f_{1}(x) d x+(1-\alpha) \int_{0}^{\infty} x^{r} f_{2}(x) d x,
$$

where $f_{1}(x)$ and $f_{2}(x)$ are defined as in equations (2). The first. five theoretical moments about the origin of (1) follow as 


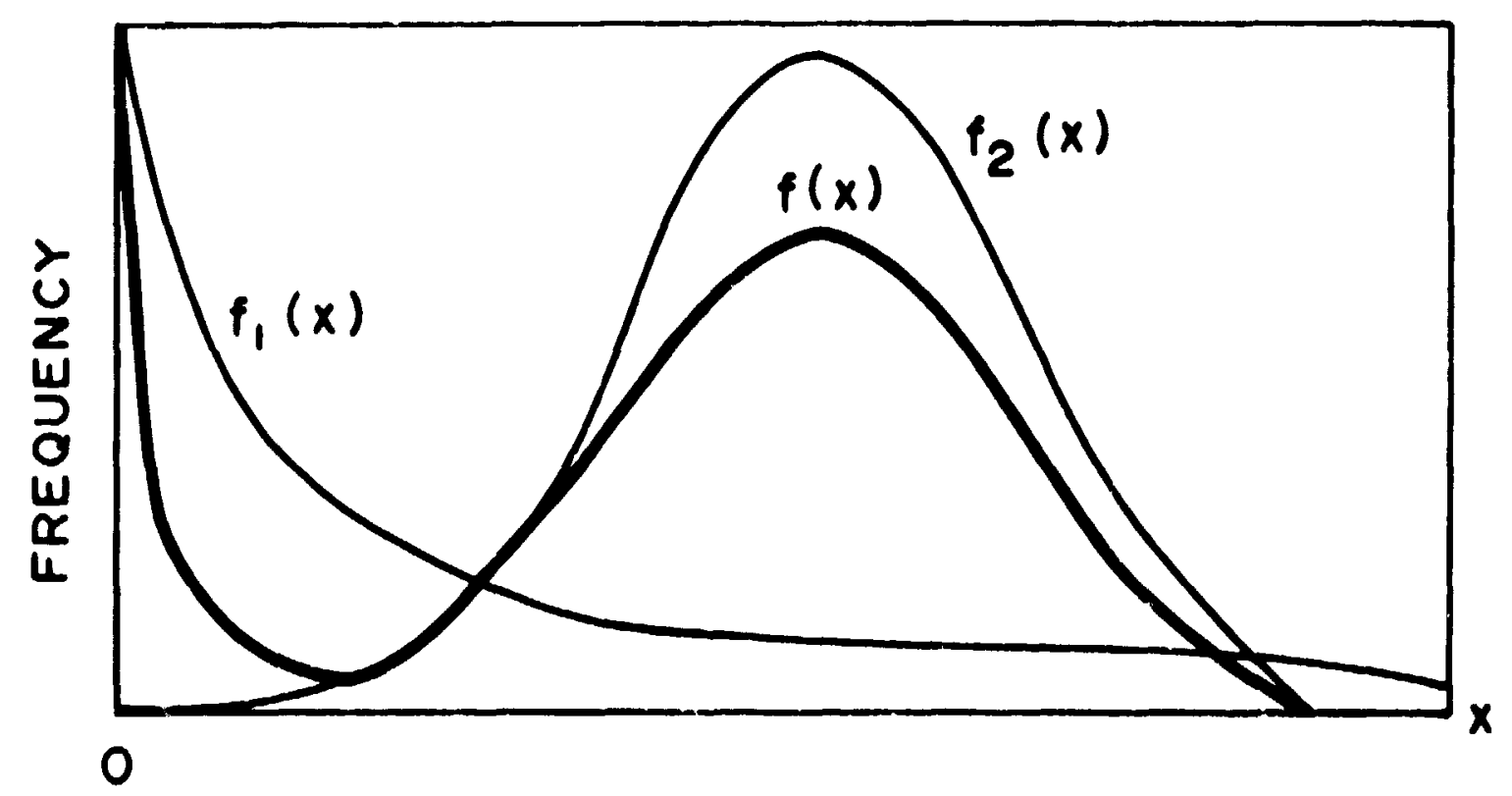

MIXED WEIBULL PROBABILITY DENSITY FUNCTION FIGURE 1

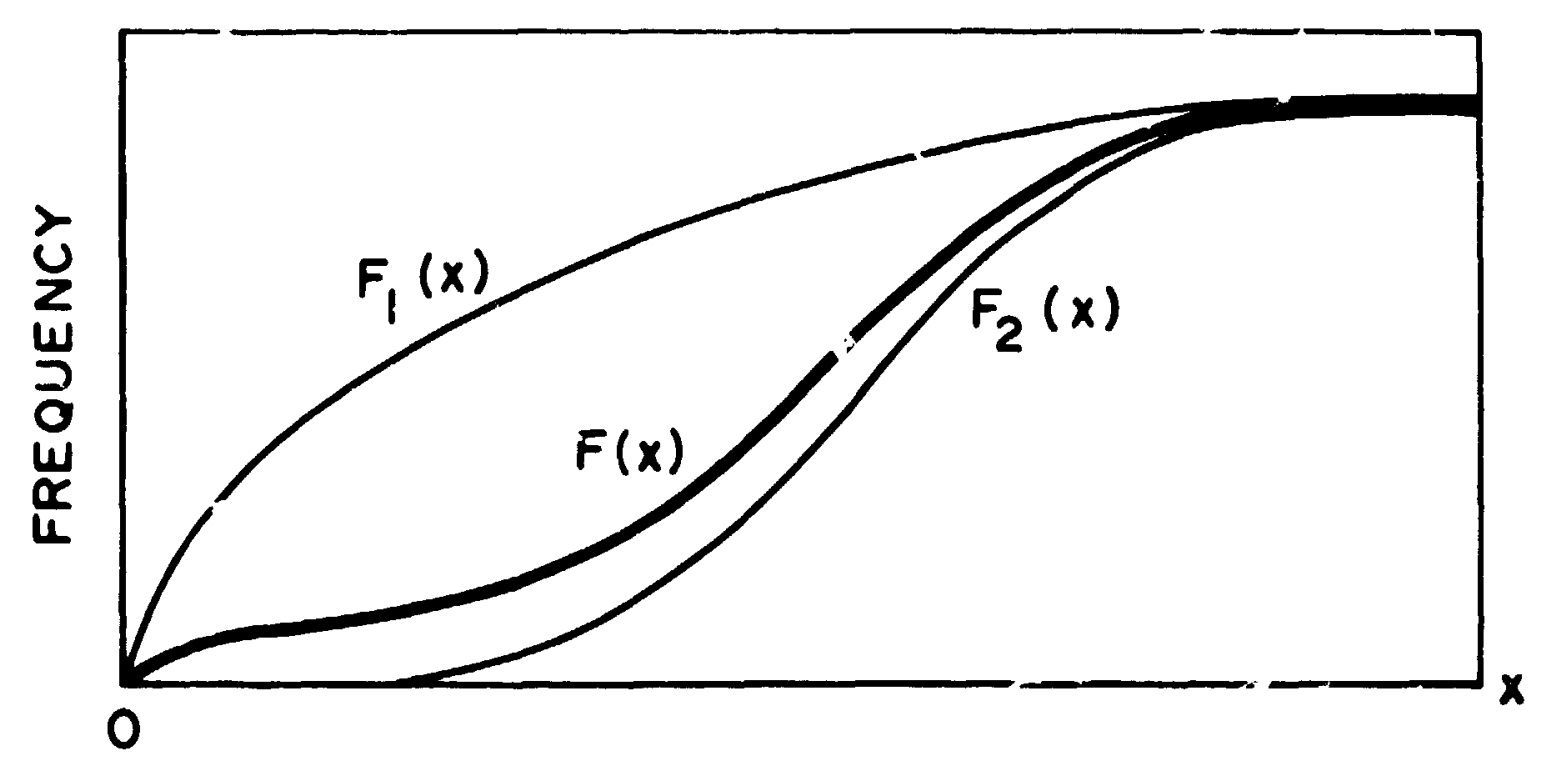

MIXED WEIBULL CUMULATIVE DISTRIBUTION FUNCTION FIGURE 2 


$$
\begin{aligned}
& \mu_{1}^{\prime}=\alpha \theta_{1}^{1 / \gamma_{1}} \Gamma\left(\frac{1}{\gamma_{1}}+1\right)+(1-\alpha) \theta_{2}^{1 / \gamma_{2}} \Gamma\left(\frac{1}{\gamma_{2}}+1\right) \\
& \mu_{2}^{\prime}=\alpha \theta_{1}^{2 / \gamma_{1}} \Gamma\left(\frac{2}{\gamma_{1}}+1\right)+(1-\alpha) \theta_{2}^{2 / \gamma_{2}} \Gamma\left(\frac{2}{\gamma_{2}}+1\right) \\
& \mu_{3}^{\prime}=\alpha \theta_{1}^{3 / \gamma_{1}} \Gamma\left(\frac{3}{\gamma_{1}}+1\right)+(1-\alpha) \theta_{2}^{3 / \gamma_{2}} \Gamma\left(\frac{3}{\gamma_{2}}+1\right) \\
& \mu_{4}^{\prime}=\alpha \theta_{1}^{4 / \gamma_{1}} \Gamma\left(\frac{4}{\gamma_{1}}+1\right)+(1-\alpha) \theta_{2}^{4 / \gamma_{2}} \Gamma\left(\frac{4}{\gamma_{2}}+1\right) \\
& \mu_{5}^{\prime}=\alpha \theta_{1}^{5 / \gamma_{1}} \Gamma\left(\frac{5}{\gamma_{1}}+1\right)+(1-\alpha) \theta_{2}^{5 / \gamma_{2}} \Gamma\left(\frac{5}{\gamma_{2}}+1\right)
\end{aligned}
$$

where $\Gamma$ is the gamma function, 1.e.,

$$
\Gamma(k)=\int_{0}^{\infty} y^{k-1} e^{-y} d y .
$$

Employing the technique of equating population moments to corresponding sample moments, the set of equations (6) becomes

$$
\begin{aligned}
& \mathrm{m}_{1}^{\prime}=\alpha \theta_{1}^{1 / \gamma_{1}} \Gamma\left(\frac{1}{\gamma_{1}}+1\right)+(1-\alpha) \theta_{2}^{1 / \gamma_{2}} \Gamma\left(\frac{1}{\gamma_{2}}+1\right) \\
& \mathrm{m}_{2}^{\prime}=\alpha \theta_{1}^{2 / \gamma_{1}} \Gamma\left(\frac{2}{\gamma_{1}}+1\right)+(1-\alpha) \theta_{2}^{2 / \gamma_{2}} \Gamma\left(\frac{2}{\gamma_{2}}+1\right) \\
& \mathrm{m}_{3}^{\prime}=\alpha \theta_{1}^{3 / \gamma_{2}} \Gamma\left(\frac{3}{\gamma_{1}}+1\right)+(1-\alpha) e_{<}^{3 / \gamma_{2}} \Gamma\left(\frac{3}{\gamma_{2}}+1\right) \\
& \mathrm{m}_{4}^{\prime}=\alpha \theta_{1}^{4 / \gamma_{1}} \Gamma\left(\frac{4}{\gamma_{1}}+1\right)+(1-\alpha) \theta_{2}^{4 / \gamma_{2}} \Gamma\left(\frac{4}{\gamma_{2}}+1\right) \\
& \mathrm{m}_{5}^{\prime}=\alpha \theta_{1}^{5 / \gamma_{1}} \Gamma\left(\frac{5}{\gamma_{1}}+1\right)+(1-\alpha) \theta_{2}^{5 / \gamma_{2}} \Gamma\left(\frac{5}{\gamma_{2}}+1\right)
\end{aligned}
$$

5 
where $m_{i}^{\prime}(i=1,2, \ldots, 5)$ is the $i$ th noncentral moment of the sample.

The set of equations (7) is s system of five equations which must be solved simultaneously for estimates of the five parameters $\alpha, \theta_{1}$, $\partial_{2}, \gamma_{1}$ and $\gamma_{2}$. For convenience in handling these equations, we wiil make the following transformations where necessary in this paper. (This notation will be used unless stated otherwise.)

Let

$$
\begin{aligned}
& v=\theta_{1}^{1 / \gamma_{1}} \quad \theta_{1}=\Gamma\left(\frac{1}{\gamma_{1}}+1\right) \quad \psi_{1}=\Gamma\left(\frac{1}{\gamma_{2}}+1\right) \\
& \mathrm{n}=\theta_{2}^{1 / \gamma_{2}} \quad \beta_{2}=\Gamma\left(\frac{2}{\gamma_{1}}+1\right) \quad \psi_{2}=\Gamma\left(\frac{2}{\gamma_{2}}+1\right) \\
& \beta_{3}=\Gamma\left(\frac{3}{\gamma_{1}}+1\right) \quad \psi_{3}=\Gamma\left(\frac{3}{\gamma_{2}}+1\right) .
\end{aligned}
$$

Thus, the first three equations of (7) become

$$
\begin{aligned}
& \mathrm{m}_{1}^{\prime}=\alpha v \beta_{1}+(1-\alpha) \mathrm{n} \psi_{1} \\
& \mathrm{~m}_{2}^{\prime}=\alpha v^{2} \beta_{2}+(1-\alpha) \mathrm{n}^{2} \psi_{2} \\
& \mathrm{~m}_{3}^{\prime}=\alpha v^{3} \beta_{3}+(1-\alpha) \mathrm{n}^{3} \psi_{3} .
\end{aligned}
$$

Solving (9) for $v$, substituting this expression into (10), and then solving for $\mathrm{n}$ yields

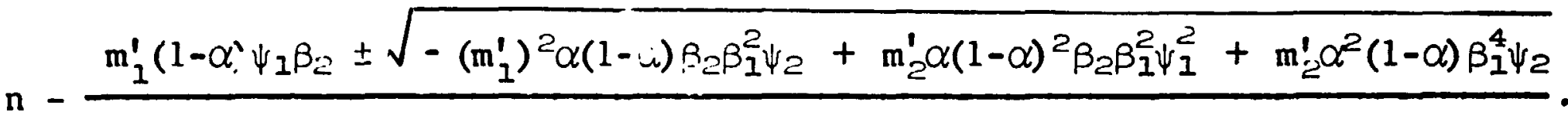

$$
(1-\alpha)^{2} \psi_{1}^{2} \beta_{2}+\alpha(1-\alpha) \beta_{1}^{2} \psi_{2}
$$


Substituting the expression for $\mathrm{n}$ from (12) back into (9) and solving for $v$ gives

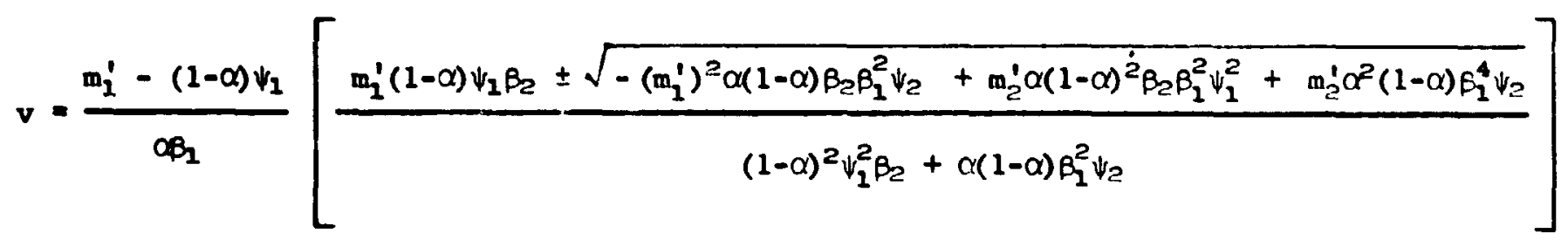

Upon substituting the expression for $n$ from (12) and the expression for $v$ from (13) into equation (11), we have one equation in the three unknowns $\alpha, \gamma_{1}$ and $\gamma_{2}$. At this point, it is obvious that exp!icit expressions for the unknown parameters $\alpha, \gamma_{1}$ and $\gamma_{2}$ carnot be obtained. Therefore, it is suggested that the following procedure be used to obtain a graphical estimate of $\alpha$, the proportionality parameter. This method is essentially that of KaO [6] and is based upon the fact that a simple Weiblil cumulative distribution becomes a straight 1 ine in $1 n$ versus ${ }^{1} n-1 n$ coordinates. This method of estimating $\alpha$ will produce a relatively small error in the estimating procedure since $\alpha$ is limited to the range $0<\alpha<1$.

(1) Plot the sample cum'ilative distribution function for the mixed data on special $1 n$ versus $1 n-\ln$ paper and visually fit a curve (called Weibull plot) among these points.*

(2.) Starting at each end of the Weibull plot, draw two tangent lines and denote them by $\widetilde{\alpha F_{I}}$ and $\overline{(1-\alpha) F_{2}}$ which are estimates of $\alpha F_{1}(x)$ and $(1-\alpha) F_{2}(x)$, respectively.

(3) At the intersection of $\overline{(1-\alpha) F_{2}}$ with the upper borderline drop a vertical line whose intersection with $\widehat{\alpha F_{I}}$ as read from the percent scale gives our estimate of $\alpha$.

See Figure 4 under Section IV for an illustration of this method.

Once an estimate of $\alpha$ has been determined graphically, solve equation (11) for $\gamma_{1}$ and $\gamma_{2}$ by the following iterative procedure. This procedure is taken from Cohen [1] and is a modified Newton-Raphson method.

Assume a value for $\gamma_{1}$ and solve equation (11) for a first approximation to $\gamma_{2}$. These first approximations can be substituted into (12) and (13) to obtain first approximations to $\theta_{1}$ and $\theta_{2}$. The first set of approximations are then introduced into the fourth equation of (6) to approximate the fourth non-central theoretical moment, $\mu_{t_{i}^{\prime}}^{\prime}$.

*Special Weibull graph paper is available from Cornell University, Ithaca, New York. 
Let $\gamma_{1(i)}$ denote the ith approximation to $\gamma_{1}$ and let $\mu_{4(i)}^{\prime}$ denote the ith approximation (corresponding to $\gamma_{1(i)}$ ) to $\mu_{4}^{\prime}$. It should be relatively easy to find approximations $\gamma_{1(i)}$ and $\gamma_{1(i+1)}$ such that the sample moment $m_{4}^{\prime}$ is in the interval $\left[\mu_{4(i)}^{\prime}, \mu_{4(i+1)}^{\prime}\right]$. Once the interval between $\gamma_{I(i)}$ and $\gamma_{I(i+1)}$ has been narrowed sufficiently, the required estimate $\gamma_{1}$ can be obtained by a simple linear interpolation as indicated below.

\begin{tabular}{|c|c|}
\hline$\gamma_{2}$ & $\mu_{4}^{\prime}$ \\
\hline$\gamma_{1(i)}$ & $\mu_{4(i)}^{\prime}$ \\
$\gamma_{1}$ & $\mathrm{~m}_{4}^{\prime}$ \\
$\gamma_{2(i+1)}$ & $\mu_{4(i+1)}^{\prime}$ \\
\hline
\end{tabular}

The required estimate of $\gamma_{2}$ can subsequently be obtained from equation (11). Once $\gamma_{1}$ and $\gamma_{2}$ have been determined by equation (11), estimates for $\mathrm{O}_{1}$ and $\mathrm{O}_{2}$ are obtained from equations (13) and (12), respectively.

Unfortunately, the quadratic solutions in equations (12) and (13) result in more than one set of estimates. The problem of non-unique sets of estimates was considered by Karl Pearson [15] and A. C. Cohen, Jr. [1] in connection with mixtures of two norma $i$ distributions. Pearson suggested choosing the set of estimates which gives closest agreement between the sixth sample moment and the sixth theoretical moment after equating the first five sample moments to the corresponding theoretical moments. This procedure is followed for all acceptable sets of estimates.

Cohen [1] suggests, as an alternate procedure for resolving the problem of multiple sets of estimates, that we might choose the set of estimates which produces the smallest Chi-Square Index of Dispersion when observed frequencies are compared with expected frequencies.

In the general case of estimation considered here, we are concerned with a mixture of two Weibull distributions where the proportionality lactor $\alpha$ is estimated graphically from the cumulative frequencies and the four remaining parameters are estimated by equating the first four sample moments to corresponding theoretical moments. When confronted with more than one set of acceptable estimates, we adopt Pearson's suggested procodure and choose the set which produces the closest agreement between 
the fifth noncentral moment of the sample $\mathrm{m}_{5}^{\prime}$ and the theoretical moment $\mu_{5}^{\prime} g$ iven by the final equation of $(6)$.

The calculations described above may be carried out iteratively with relative ease using the computer program included in this paper as an Appendix. First approximations to initiate the iterative process may be obtained using a graphical method such as that of Kao [6].

\section{ESTIMATION IN SPECIAL CASES}

A number of speci 1 cases that may be of practical value in which certain parameters are known or are restricted in some manner are considered.

1. $\theta_{1}$ known. With $\theta_{1}$ known, we must estimate the parameters $\alpha$, $\theta_{2}, \gamma_{1}$ and $\gamma_{2}$ only. If we let $v=\theta_{1}^{1 / \gamma_{1}}$, equations $(9),(10)$ and (11) become

$$
\begin{aligned}
& m_{1}^{\prime}=\alpha \theta_{1}^{1 / \gamma_{1}} \beta_{1}+(1-\alpha) n \psi_{1}, \\
& m_{2}^{\prime}=\alpha \theta_{1}^{2 / \gamma_{1}} \beta_{2}+(1-\alpha) n^{2} \psi_{2}, \\
& m_{3}^{\prime}=\alpha \theta_{1}^{3 / \gamma_{1}} \beta_{3}+(1-\alpha) n^{3} \psi_{3} .
\end{aligned}
$$

Solving (14) for $\alpha$ gives

$$
\alpha=\frac{m_{1}^{\prime}-n \psi_{1}}{\theta_{1}^{1 / \gamma_{1}} \beta_{1}-n \psi_{1}}
$$

Inserting this expression for $\alpha$ into equation (15) and solving for $n$, we obtain after considerable algebraic manipulation

$=\frac{v^{2} v_{2} \beta_{2}-z_{2}^{\prime} v_{1} \pm \sqrt{\left(m_{2}^{\prime}\right)^{2} v_{1}^{2}-2 m_{2}^{\prime} v^{2} v_{1}^{2} \beta_{2}+v^{4} v_{1}^{2} \beta_{2}^{2}-4 m_{1}^{\prime} v^{3} \beta_{1} \beta_{2} v_{2}+4\left(m_{1}^{\prime}\right)^{2} v^{2} \beta_{2} v_{2}+4 m_{2}^{\prime} v^{2} \beta_{2}^{2} v_{2}-4 m_{2}^{\prime} m_{2}^{\prime} v \beta_{1} v_{2}}}{2 v \beta_{1} v_{2}-2 m_{1}^{\prime} v_{2}}$ 
Substituting this expression for $\mathrm{n}$ back into (17) gives

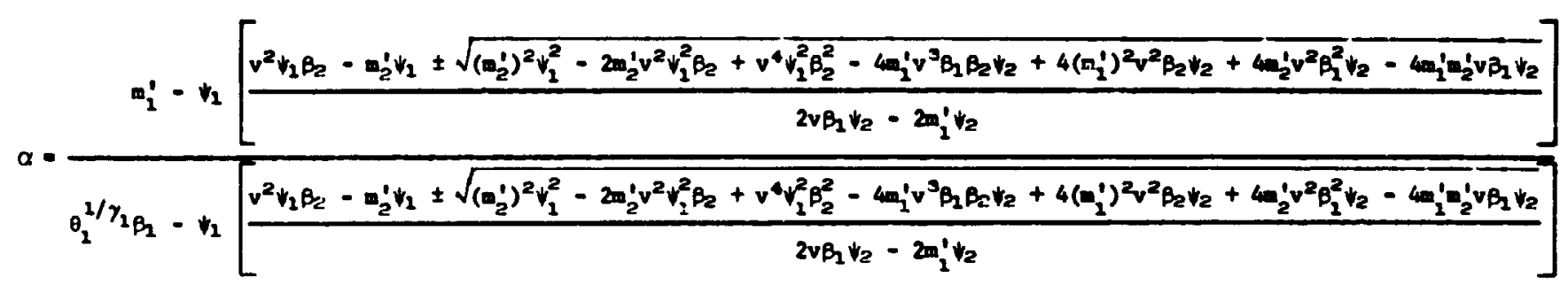

Now, upon substituting the expression for $\mathrm{n}$ from (18) and the expression for $\alpha$ lrom (19) into equation (16), we have an equation in the two unknowns $\gamma_{1}$ and $\gamma_{2}$ which may be solved by the iterative procedure described in the general case. Once $\gamma_{1}$ and $\gamma_{2}$ have been determined, we obtain our estimates for $\theta_{2}$ and $\alpha$ from equations (18) and (19), respectively. As in the general case, the positive and negative roots resulting from the quadratic solution in (18) unfortunately results in more than one set of estimates. As before, it is suggested that the set of estimates which gives the closest agreement between the fifth noncentral moment of the sample and the corresponding "fitted" compound curve be used.

2. $\theta_{2}$ known, With $\theta_{2}$ known we need only estimate $\alpha, \theta_{1}, \gamma_{1}$ and $\gamma_{2}$. If we let $n=\theta_{2}^{1 / \gamma_{2}}$, equations (9), (10) and (11) become

$$
\begin{aligned}
& \mathrm{m}_{1}^{\prime}=\alpha v \beta_{1}+(1-\alpha) \theta_{2}^{1 / \gamma_{2}} \psi_{1}, \\
& \mathrm{~m}_{2}^{\prime}=\alpha v=\beta_{2}+(1-\alpha) \theta_{2}^{2 / \gamma_{2}} \psi_{2}, \\
& \mathrm{~m}_{3}^{\prime}=\alpha v^{3} \beta_{3}+(1-\alpha) \theta_{2}^{3 / \gamma_{2}} \psi_{3} .
\end{aligned}
$$

Solving (20) for $\alpha$ gives

$$
\alpha=\frac{m_{1}^{\prime}-0_{2}^{1 / \gamma_{2}} \psi_{1}}{v \beta_{1}-0_{2}^{1 / \gamma_{2}} \psi_{1}} .
$$


Inserting this expression for $\alpha$ into equation (21) and solving for $v$, we obtain after considerable algebraic manipulation

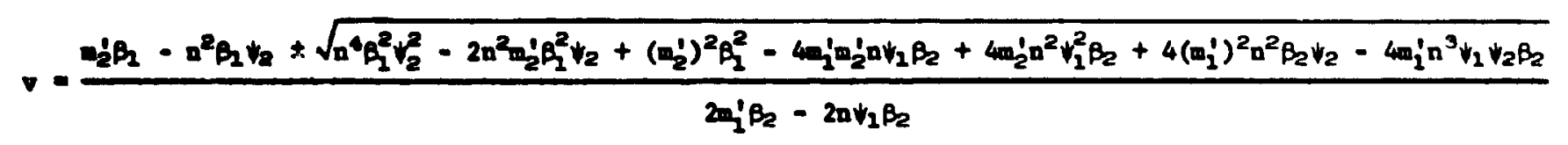

Substituting this expression for $v$ back into (23) gives

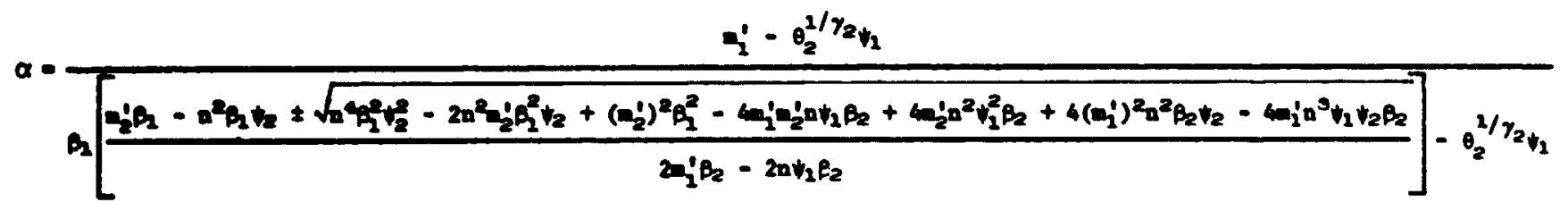

Now, upon substituting the expression for $v$ from (24) and the expression for $\alpha$ from (25) into equation (22), we obtain an equation in the two unknowns $\gamma_{1}$ and $\gamma_{2}$ which may be solved by the iterative process described previously. Once $\gamma_{1}$ and $\gamma_{2}$ are determined, we obtain our estimates for $\theta_{1}$ and $\alpha$ from equations (24) and (25). As in case $1, \theta_{1}$ known, the positive and negative roots resulting from the quadratic solution in (24) gives more than one set of estimates. Again, it is suggested that we choose the set of estimates which gives the closest agreement between the fifth noncentral moment of the sample and the corresponding "fitted" compound curve.

3. $\gamma_{1}$ known. If $\gamma_{1}$ is known we must estimate $\alpha, \theta_{1}, \theta_{2}$ and $\gamma_{2}$ only. Solving equation (9) for $v$ gives

$$
v=\frac{m_{1}^{\prime}-(1-\alpha) n \psi_{1}}{\alpha \beta_{1}}
$$

Inserting this expression for $v$ iuto equation (10) and solving fo: ?, we obtain

$$
\mathbf{n}=\frac{\mathrm{m}_{1}^{\prime}(1-\alpha) \psi_{1} \beta_{2} \pm \sqrt{-\left(\mathrm{m}_{1}^{\prime}\right)^{2} \alpha(1-\alpha) \beta_{2} \beta_{1}^{2} \psi_{2}+\mathrm{m}_{2}^{\prime} \alpha(1-\alpha)^{2} \beta_{2} \beta_{1}^{2} \psi_{1}^{2}+\mathrm{m}_{2}^{\prime} \alpha^{2}(1-\alpha) \beta_{1}^{4} \psi_{2}^{2}}}{(1-\alpha)^{2} \psi_{1}^{2} \beta_{2}+\alpha(1-\alpha) \beta_{1}^{2} \psi_{2}} .
$$


Substituting this expression for $n$ back into (26) gives

$v=\frac{m_{1}^{\prime}-(1-\alpha) \psi_{1}}{\alpha \beta_{1}}\left[\frac{m_{1}^{\prime}(1-\alpha) \psi_{1} \beta_{2} \pm \sqrt{-\left(m_{1}^{\prime}\right)^{2} \alpha(1-\alpha) \beta_{2} \beta_{1}^{2} \psi_{2}+m_{2}^{\prime} \alpha(1-\alpha)^{2} \beta_{2} \beta_{1}^{2} \psi_{1}^{2}+m_{2}^{\prime} \alpha^{2}(1-\alpha) \beta_{1}^{4} \psi_{2}}}{(1-\alpha)^{2} \psi_{1}^{2} \beta_{2}+\alpha(1-\alpha) \beta_{1}^{2} \psi_{2}}\right]$

Upon substituting the expression for $n$ from (27) and the expression for $v$ from (28) into equation (11), we obtain an equation in the two unknowns $\alpha$ and $\gamma_{2}$ which may be solved by the iterative procedure described in the general case. With $\alpha$ and $\gamma_{2}$ determined we may solve equations (27) and (28) for $\theta_{2}$ and $\theta_{1}$, respectively. As before, the positive and negative roots which result from the quadratic solution in (27) give more than one set of estimates. Again, we choose the set of estimates which gives the closest agreement to the fifth noncentral moment of the sample.

An alternate method for estimation in this case would be to estimate $\alpha$ graphically as in the general case and then solve equation (11) for $\gamma_{2}$ after the substitution of the expression for $n$ from (27) and the expression for $v$ from (28) into equation (11). As before, $\theta_{2}$ and $\theta_{1}$ would then be obtained from. equations (27) and (28).

4. $\gamma_{2}$ known. With $\gamma_{2}$ known, we must estimate $\alpha, \theta_{1}, \theta_{2}$ and $\gamma_{1}$ only. As in case $3, \gamma_{1}$ known, solving equation (9) for $v$ gives

$$
\mathrm{v}=\frac{\mathrm{m}_{1}^{\prime}-(1-\alpha) \mathrm{n} \psi_{1}}{\alpha \beta_{1}}
$$

Inserting this expression for $\mathrm{v}$ into equation (10) and solving for $\mathrm{n}$, we obtain

$\mathrm{n}=\frac{\mathrm{m}_{1}^{\prime}(1-\alpha) \psi_{1} \beta_{2} \pm \sqrt{-\left(\mathrm{m}_{1}^{\prime}\right)^{2} \alpha(1-\alpha) \beta_{2} \beta_{1}^{2} \psi_{2}+\mathrm{m}_{2}^{\prime} \alpha(1-\alpha)^{2} \beta_{2} \beta_{1}^{2} \psi_{1}^{2}+\mathrm{m}_{2}^{\prime} \alpha^{2}(1-\alpha) \beta_{1}^{4} \psi_{2}}}{.}$

$$
(1-\alpha)^{2} \psi_{1}^{2} \beta_{2}+\alpha(1-\alpha) \beta_{1}^{2} \psi_{2}
$$


When we substitute this expression for $n$ back into (29), we get

$$
v=\frac{m_{1}^{\prime}-(1-\infty) \psi_{2}}{\beta_{1}}\left[\frac{m_{1}^{\prime}(1-\alpha) \psi_{1} \beta_{2} \pm \sqrt{-\left(m_{1}^{\prime}\right)^{2} \alpha(1-\alpha) \beta_{2} \beta_{2}^{2} \psi_{2}+m_{2}^{\prime} \alpha(1-\alpha)^{2} \beta_{2} \beta_{1}^{2} \psi_{1}^{2}+m_{2}^{\prime} \alpha^{2}(1-\alpha) \beta_{1}^{4} \psi_{2}}}{(1-\alpha)^{2} \psi_{1}^{2} \beta_{2}+\alpha(1-\alpha) \beta_{1}^{2} \psi_{2}}\right]
$$

Upon inserting the expression for $n$ from (30) and the expression for $v$ from (31) into equation (11), we obtain an equation in the two unknowns $\alpha$ and $\gamma_{1}$ which may be solved by the iterative procedure described previously. With $\alpha$ and $\gamma_{1}$ determined in this manner, we now solve equations (30) and (31) for $\theta_{2}$ and $\theta_{1}$, respectively. As before, we choose the set of estimates which gives the closest agreement between the fifth noncentral moment of the sample and the corresponding "fitted" compound curve.

As in case 3, $\gamma_{1}$ known, an alternate method for estimation would be to estimate $\alpha$, the proportionality parameter graphically, and then solve equation (11) directly for $\gamma_{1}$ after the substitution of the expression for $n$ from (30) and the expression for $v$ from (31) into equation (11).

5. $\gamma_{1}=1$. This is a special case of case $3, \gamma_{1}$ known. Thus, case 3 is reduced to mixing an exponential distribution with a Weloull distribution where $f_{1}(x)$ in equations $(2)$ is an exponential distribution and $f_{2}(x)$ is a Weibull distribution. We need only estimate $\alpha, \gamma_{2}, \theta_{1}$ and $\theta_{2}$.

With $\gamma_{1}=1$, equations (9), (10) and (11) become

$$
\begin{aligned}
& m_{1}^{\prime}=\alpha v+(1-\alpha) n \psi_{1} \\
& m_{2}^{\prime}=20 v^{2}+(1-\alpha) n^{2} \psi_{2} \\
& m_{3}^{\prime}=60 v^{3}+(1-\alpha) n^{3} \psi_{3},
\end{aligned}
$$

where the only change in notation from previous cases is $v=\theta_{1}$. 
Solving equation (32) for $v$ gives

$$
v=\frac{m_{1}^{\prime}-(1-\alpha) n \psi_{1}}{\alpha}
$$

Substituting this expression for $v$ into equation (33) and solving for n gives

$n=\frac{2 m_{1}^{\prime}(1-\alpha) \psi_{1} \pm \sqrt{2 m_{2}^{\prime} \alpha(1-\alpha)^{2} \psi_{1}^{2}-2\left(m_{1}^{\prime}\right)^{2} \alpha(1-\alpha) \psi_{2}+m_{2}^{\prime} \alpha^{2}(1-\alpha) \psi_{2}}}{2(1-\alpha)^{2} \psi_{1}^{2}+\alpha(1-\alpha) \psi_{2}}$.

Inserting this expression for $n$ back into (35) gives

$v=\frac{m_{1}^{\prime}}{\alpha}-\psi_{1}\left[\frac{2 m_{1}^{\prime}(1-\alpha) \psi_{1} \pm \sqrt{2 m_{2}^{\prime} \alpha(1-\alpha)^{2} \psi_{1}^{2}-2\left(m_{1}^{\prime}\right)^{2} \alpha(1-\alpha) \psi_{2}+m_{2}^{\prime} \alpha^{2}(1-\alpha) \psi_{2}}}{2 \alpha(1-\alpha) \psi_{1}^{2}+\alpha^{2} \psi_{2}}\right]$.

Now, upon substituting the expression for $n$ from (36) and the expression for $v$ from (37) into equation (34), we obtain an equation in the two unknowns $\alpha$ and $\gamma_{2}$ which we may solve using the iterative procedure described in previous cases. With minor simplifications, equation (34) becomes

$m_{3}^{\prime}=6 \alpha\left[\frac{m_{1}^{\prime}}{\alpha}-\psi_{1}\left(\frac{2 m_{1}^{\prime}(1-\alpha) \psi_{1} \pm \sqrt{2 m_{2}^{\prime} \alpha(1-\alpha)^{2} \psi_{1}^{2}-2\left(m_{1}^{2}\right)^{2} \alpha(1-\alpha) \psi_{2}+m_{2}^{\prime} \alpha^{2}(1-\alpha) \psi_{2}}}{2 \alpha(1-\alpha) \psi_{1}^{2}+\alpha^{2} \psi_{2}}\right)\right]^{3}+$

$$
(1-\alpha) \psi_{3}\left[\frac{2 m_{1}^{\prime}(1-\alpha) \psi_{1} \pm \sqrt{2 m_{2}^{\prime} \alpha(1-\alpha)^{2} \psi_{1}^{2}-2\left(m_{1}^{\prime}\right)^{2} \alpha(1-\alpha) \psi_{2}+m_{2}^{\prime} \alpha^{2}(1-\alpha) \psi_{2}}}{2(1-\alpha)^{2} \psi_{1}^{2}+\alpha(1-\alpha) \psi_{2}}\right]^{3}
$$


Once $\alpha$ and $\gamma_{2}$ have been determined from equation (38) we may obtain our estimates for $\theta_{2}$ and $\theta_{1}$ from equations (36) and (37), respectively.

As in the other cases, we choose the set of estimates which gives the closest agreement between the fifth noncentral moment of the sample and the corresponding "fitted" compound curve.

An alternate method of estimation would be to estimate $\alpha$ graphically as in the general case and then solve equation (38) directly for $\gamma_{2}$. As above, estimates for $\theta_{2}$ and $\theta_{1}$ would then be obtained from equations (36) and (37).

6. $\gamma_{1}=\gamma_{2}=$ unknown. Changing our notation slightly, we will let $\gamma_{1}=\gamma_{2}=\gamma$. Thus, we must estimate $\alpha, \gamma, \theta_{1}$ and $\theta_{2}$. Now, let

$$
\begin{array}{ll}
v=\theta_{1}^{1 / \gamma} & \beta_{1}=\Gamma\left(\frac{1}{\gamma}+1\right) \quad \beta_{3}=\Gamma\left(\frac{3}{\gamma}+1\right) \\
r_{1}=\theta_{2}^{1 / \gamma} & \beta_{2}=\Gamma\left(\frac{2}{\gamma}+1\right) .
\end{array}
$$

With this notation, equations (9), (10) and (11) become

$$
\begin{aligned}
& \mathrm{m}_{1}^{\prime}=\alpha \mathbf{v} \beta_{1}+(1-\alpha) \mathrm{n} \beta_{1} \\
& \mathbf{m}_{2}^{\prime}=\alpha v^{2} \beta_{2}+(1-\alpha) n^{2} \beta_{2} \\
& m_{3}^{\prime}=\alpha v^{3} \beta_{3}+(1-\alpha) n^{3} \beta_{3} .
\end{aligned}
$$

As before, solving equation (39) for $v$ gives

$$
v=\frac{m_{1}^{\prime}-(1-\alpha) n \beta_{1}}{\alpha \beta_{1}}
$$


Inserting this expression for $v$ into equation (40) and subsequently solving for $n$, we have

$$
n=\frac{m_{1}^{\prime}(1-\alpha) \beta_{2} \pm \sqrt{(1-\alpha) \beta_{2} \alpha\left[m_{2}^{\prime} \beta_{1}^{2}-\left(m_{1}^{\prime}\right)^{2} \beta_{2}\right]}}{(1-\alpha) \beta_{1} \beta_{2}}
$$

Substituting this expression for $\mathrm{n}$ back into (42) gives

$$
v=\frac{m_{1}^{\prime}}{\alpha \beta_{1}}-\frac{m_{1}^{\prime}(1-\alpha) \beta_{2} \pm \sqrt{\alpha(1-\alpha) \alpha\left[m_{2}^{\prime} \beta_{1}^{2}-\left(m_{1}^{\prime}\right)^{2} \beta_{2}\right]}}{\alpha \beta_{1} \beta_{2}}
$$

Upon substituting the expression for $n$ from (43) and the expression for $v$ from (44) into equation (41), we obtain an equation in the two unknowns $\alpha$ and $\gamma$ which may be solved by the iterative process described in the general case. We may now solve equations (43) and (44) for $\theta_{2}$ and $\theta_{1}$. As in the other cases, the quadratic solution in equation (43) results in more than one set of estimates. Again, we use the set of estimates which gives the closest agreement to the fifth noncentral moment of the sample.

As before, an alternate solution would be to estimate $\alpha$ graphically and then solve the resulting equation (41) directly for $\gamma$.

In the event that $\alpha$ is known in advance of sampling we may solve equation (41) directly for $\gamma$ and subsequently obtain $\theta_{2}$ and $\theta_{1}$ from (43) and (44).

7. $\gamma_{1}=\gamma_{2}=$ known. If we let $\gamma_{1}=\gamma_{2}=\gamma$, the first three equations of (7) become

$$
\begin{aligned}
& \mathrm{m}_{1}^{\prime}=\alpha \theta_{1}^{1 / \gamma} \Gamma\left(\frac{1}{\gamma}+1\right)+(1-\alpha) \theta_{2}^{1 / \gamma} \Gamma\left(\frac{1}{\gamma}+1\right) \\
& \mathrm{m}_{2}^{\prime}=\alpha 0_{1}^{2 / \gamma} \Gamma\left(\frac{2}{\gamma}+1\right)+(1-\alpha) \theta_{2}^{2 / \gamma} \Gamma\left(\frac{2}{\gamma}+1\right) \\
& m_{3}^{\prime}=\alpha 0_{1}^{3 / \gamma} \Gamma\left(\frac{3}{\gamma}+1\right)+(1-\alpha) \theta_{2}^{3 / \gamma} \Gamma\left(\frac{3}{\gamma}+1\right) .
\end{aligned}
$$


Thus, we must estimate $\alpha, \theta_{1}$ and $\theta_{2}$ only. For simplification, we will let

$$
c_{j}=\frac{m_{1}^{\prime}}{\Gamma\left(\frac{1}{\gamma}+1\right)}
$$

Now, equations (45), (46) and (47) become

$$
\begin{aligned}
& \alpha \theta_{1}^{2 / \gamma}+(1-\alpha) \theta_{2}^{1 / \gamma}=c_{1} \\
& \alpha \theta_{1}^{2 / \gamma}+(1-\alpha) \theta_{2}^{2 / \gamma}=c_{2} \\
& \alpha \theta_{1}^{3 / \gamma}+(1-\alpha) \theta_{2}^{3 / \gamma}=c_{3} .
\end{aligned}
$$

Solving equation (48) for $\alpha$ we have

$$
\alpha=\frac{c_{1}-\theta_{2}^{1 / \gamma}}{\theta_{1}^{1 / \gamma}-\theta_{2}^{1 / \gamma}} \text {. }
$$

Substituting the expression for $\alpha$ from (51) into (49) and (50), we have after considerable alg tbraic manipulation the equations

$$
\begin{gathered}
\theta_{1}^{1 / \gamma} \theta_{2}^{1 / \gamma}=c_{2}\left(\theta_{1}^{1 / \gamma}+\theta_{2}^{1 / \gamma}\right)-c_{2} \\
\theta_{1}^{1 / \gamma} \theta_{2}^{1 / \gamma}\left(\theta_{1}^{1 / \gamma}+\theta_{2}^{1 / \gamma}\right)=c_{1}\left(\theta_{1}^{2 / \gamma}+\theta_{1}^{2 / \gamma} \theta_{2}^{1 / \gamma}+\theta_{2}^{2 / \gamma}\right)-c_{3} .
\end{gathered}
$$

Inserting the expression for $\theta_{1}^{2 / \gamma} \theta_{2}^{2 / \gamma}$ from (52) into the left side of equation (53), we obtain after 8 implifying

$$
\theta_{1}^{1 / \gamma} \theta_{2}^{1 / \gamma} c_{1}-c_{2}\left(\theta_{1}^{1 / \gamma}+\theta_{2}^{1 / \gamma}\right)+c_{3}=0 .
$$


Solving (54) for $\theta_{1}^{2 / \gamma}$ gives

$$
\theta_{1}^{1 / \gamma}=\frac{\theta_{2}^{1 / \gamma} c_{2}-c_{3}}{e_{2}^{1 / \gamma} c_{1}-c_{2}}
$$

Substituting this expression for $\theta_{1}^{1 / \gamma}$ back into equation (52), we have after simplitication the quadratic equation in $\theta_{i}^{1 / \gamma}$

$$
\left(c_{2}-c_{1}^{2}\right)\left[\theta_{i}^{1 / \gamma}\right]^{2}-\left(c_{3}-c_{1} c_{2}\right) \sigma_{i}^{1 / \gamma}+\left(c_{1} c_{3}-c_{2}^{2}\right)=0
$$

whose solution for $\theta_{i}$ is

$0_{i}=\left[2\left(c_{1}^{c}-c_{2}\right)^{-1}\left[\left(-c_{3}+c_{1} c_{2}\right) \pm\left(c_{3}^{2}-6 c_{1} c_{2} c_{3}-3 c_{1}^{2} c_{2}^{2}+4 c_{1}^{3} c_{3}+4 c_{2}^{3}\right)^{1 / z}\right]\right]^{\gamma}$.

Without loss of generality, we may impose the restriction that $\theta_{1}<\theta_{2}$. Thus, we obtain $\theta_{I}$ and $\theta_{2}$ from equation (57) using the negative and positive roots, respectively. Once we have determined $\theta_{1}$ and $\theta_{2}$, we obtain our estimate for $\alpha$ from equation (51).

8. $\theta_{1}=\theta_{2}=$ known. If we let $\theta_{1}=\theta_{2}=\theta$, the first three equations of (7) become

$$
\begin{aligned}
& \mathrm{m}_{1}^{\prime}=\alpha \theta^{1 / \gamma_{1}} \beta_{1}+(1-\alpha) \theta^{1 / \gamma_{2}} \psi_{1} \\
& \mathrm{~m}_{1}^{\prime}=\alpha e^{2 / \gamma_{1}} \beta_{2}+(1-\alpha) \theta^{2 / \gamma_{2}} \psi_{2} \\
& \mathrm{~m}_{3}^{\prime}=\alpha 0^{1 / \gamma_{1}} \beta_{3}+(1-\alpha) \theta^{3 / \gamma_{2}} \psi_{3} .
\end{aligned}
$$


Thus, we must estimate the parameters $\alpha, \gamma_{1}$ and $\gamma_{2}$ only. As previously, solving (58) for $\alpha$ gives us

$$
\alpha=\frac{m_{1}^{\prime}-\theta^{1 / \gamma_{2}} \psi_{1}}{\theta^{1 / 1 / \beta_{1}}-\theta^{1 / \gamma_{Z_{1}}}}
$$

Substituting this expression for $\alpha$ into equation (59), we have after simplifying

$$
\begin{gathered}
m_{1}^{\prime}\left(\theta^{2 / \gamma_{1} B_{2}}-\theta^{2 / \gamma_{2}} \psi_{2}\right)+m_{2}^{\prime}\left(\theta^{1 / \gamma_{2}} \psi_{1}-\theta^{1 / \gamma_{1}} \beta_{1}\right) \\
-\theta^{2 / \gamma_{1}} B_{2} \theta^{1 / \gamma_{2}} \psi_{1}+\theta^{2 / \gamma_{2}} \psi_{2} \theta^{1 / \gamma_{1}} B_{1}=0 .
\end{gathered}
$$

Equation (62) is an equation in the two unknowns $\gamma_{1}$ and $\gamma_{2}$ which may be solved by the iterative procedure described for the general case of estimation. Once $\gamma_{1}$ and $\gamma_{2}$ are determined, we obtain our estimate for $\alpha$ from equation (61).

9. $\theta_{1}=\theta_{2}=$ unknown. For this special case, it is suggested that we solve for $\alpha$ graphically and subsequently follow the procedure outlined for the general case of estimation.

\section{AN ILLUSTRATIVE EXAMPLE}

To illustrate the estimation procedure outlined in this paper for the general case, we will consider a sample of 2000 observations selected from a mixed population constructed by combining two Weibull distributions with $\gamma_{1}=2.0000, \theta_{1}=10.0000, \gamma_{2}=0.8000, \theta_{2}=1.0000$ and $\alpha=0.8000$. The sample is summarized in Table $I$. For the sample selected, $m_{1}^{\prime}=2.4708$, $\mathrm{m}_{2}^{\prime}=8.6270, \mathrm{~m}_{3}^{\prime}=36.3408, \mathrm{~m}_{4}^{\prime}=174.9190$ and $\mathrm{m}_{5}^{\prime}=935.3733$. 
TABLE I

A SAMPLE OF 2000 OBSERVATIONS FROM A MIXED WEIBULL POPULATION

\begin{tabular}{|r|c|r|r|r|c|}
\hline CLASSES & CLASS MARKS & $\mathrm{f}_{1}$ & $\mathrm{f}_{2}$ & $\mathrm{f}$ & $\begin{array}{c}\text { CUMULAT IVE FREQUENCY } \\
\text { IN PER CFNT }\end{array}$ \\
\hline $0-0.5$ & 0.25 & 40 & 175 & 215 & 10.75 \\
$0.5-1.0$ & 0.75 & 113 & 78 & 191 & 20.30 \\
$1.0-1.5$ & 1.25 & 170 & 47 & 217 & 31.15 \\
$1.5-2.0$ & 1.75 & 205 & 30 & 235 & 42.90 \\
$2.0-2.5$ & 2.25 & 216 & 20 & 236 & 54.70 \\
$2.5-3.0$ & 2.75 & 206 & 14 & 220 & 65.70 \\
$3.0-3.5$ & 3.25 & 180 & 10 & 190 & 75.20 \\
$3.5-4.0$ & 3.75 & 147 & 7 & 154 & 82.90 \\
$4.0-4.5$ & 4.25 & 112 & 5 & 117 & 88.75 \\
$4.5-5.0$ & 4.75 & 80 & 4 & 84 & 92.95 \\
$5.0-5.5$ & 5.25 & 54 & 3 & 57 & 95.80 \\
$5.5-6.0$ & 5.75 & 34 & 2 & 36 & 97.60 \\
$6.0-6.5$ & 6.25 & 20 & 1 & 21 & 98.65 \\
$6.5-7.0$ & 6.75 & 11 & 1 & 12 & 99.25 \\
$7.0-7.5$ & 7.25 & 6 & 1 & 7 & 99.60 \\
$7.5-8.0$ & 7.75 & 3 & 1 & 4 & 99.80 \\
$8.0-8.5$ & 8.25 & 1 & 1 & 2 & 99.90 \\
$8.5-9.0$ & 8.75 & 1 & & 1 & 99.95 \\
$9.0-9.5$ & 9.25 & 1 & & 1 & 100.00 \\
\hline
\end{tabular}

In the above table, $\mathrm{f}_{I}=$ class frequencies from $\mathrm{f}_{1}(\mathrm{x}), \mathrm{f}_{2}=$ class frequencies from $f(x)$, and $f=$ class frequencies from the resulting mixed distribution.

Figure 3 is a graph of the compound density function and its component distributions. Notice at this point that $\gamma \leqq 1$ produces a J-shaped function while $\gamma>1$ produces a bell-shaped curve.

Employing the graphical technique described in Section I provides an estimate of $(\gamma$, the proportionality parameter, equal to 0.80 as shown on Figure 4. 
Once our estimate of $\alpha$ has been determined graphically, we solve equation (11) iteratively for first approximations to $\gamma_{2}$ and $\gamma_{2}$. Corresponding first approximations for $\theta_{1}$ and $\theta_{2}$ are obtained from equations (13) and (12), respectively. Each set of first approximations is introduced into the fourth equation of (6) to approximate the fourth noncentral theoretical moment, $\mu_{4}^{\prime}$. Each set of first approximations is also substituted into the final equation of (6) to approximate the fifth noncentral theoretical moment $\mu_{5}^{\prime}$, as suggested by Pearson [15]. The set of estimates which gives the closest agreement between the fifth noncentral moment of the sample $m_{5}^{\prime}$ and the corresponding "fitted" compound curve given by the final equation of $(6)$ is the required set of estimates. Utilizing the computer program given in the Appendix, we find that our estimate of $\gamma_{1}$ lies between 1.90 and 2.10. The corresponding estimates for the remaining parameters, $\mu_{4}^{\prime}$ and $\mu_{5}^{\prime}$, are as follows:

\begin{tabular}{|c|c|c|c|c|c|}
\hline$\gamma_{1}$ & $\gamma_{2}$ & $\theta_{1}$ & $\theta_{2}$ & $\mu_{4}^{\prime}$ & $\mu_{5}^{\prime}$ \\
\hline 1.90 & 2.30 & 11.52 & 1.29 & 343.8875 & 1926.6399 \\
2.10 & 0.80 & 6.74 & 0.82 & 64.6006 & 287.5141 \\
\hline
\end{tabular}

Our next approximation to $\gamma_{1}$ is obtained by simple 1 inear interpolation as indicated below.

\begin{tabular}{|c|c|}
\hline$\gamma_{I}$ & $\mu_{4}^{\prime}$ \\
\hline 1.90 & 343.8875 \\
2.0210 & 174.9190 \\
2.10 & 64.6006 \\
\hline
\end{tabular}

Substituting $\gamma_{1}=2.0210$ into equation (11), solving for $\gamma_{2}$ and subsequently solving equations (13) and (12) for $\theta_{1}$ and $\theta_{2}$, we obtain $\gamma_{2}=0.8339, \theta_{1}=10.1496$ and $\theta_{2}=0.9929$. Introducing this set of approximations into the final equation of (6) gives $\mu_{5}^{\prime}=935.3646$. This value of $\mu_{5}^{\prime}$ is in such close agreement with the corresponding sample moment $m_{5}^{\prime}=935.3733$ that we are justified in accepting this set of approximations as our final estimates. However, if further preciseness is desired, this iterative process may be continued to any desired degree of accuracy. 


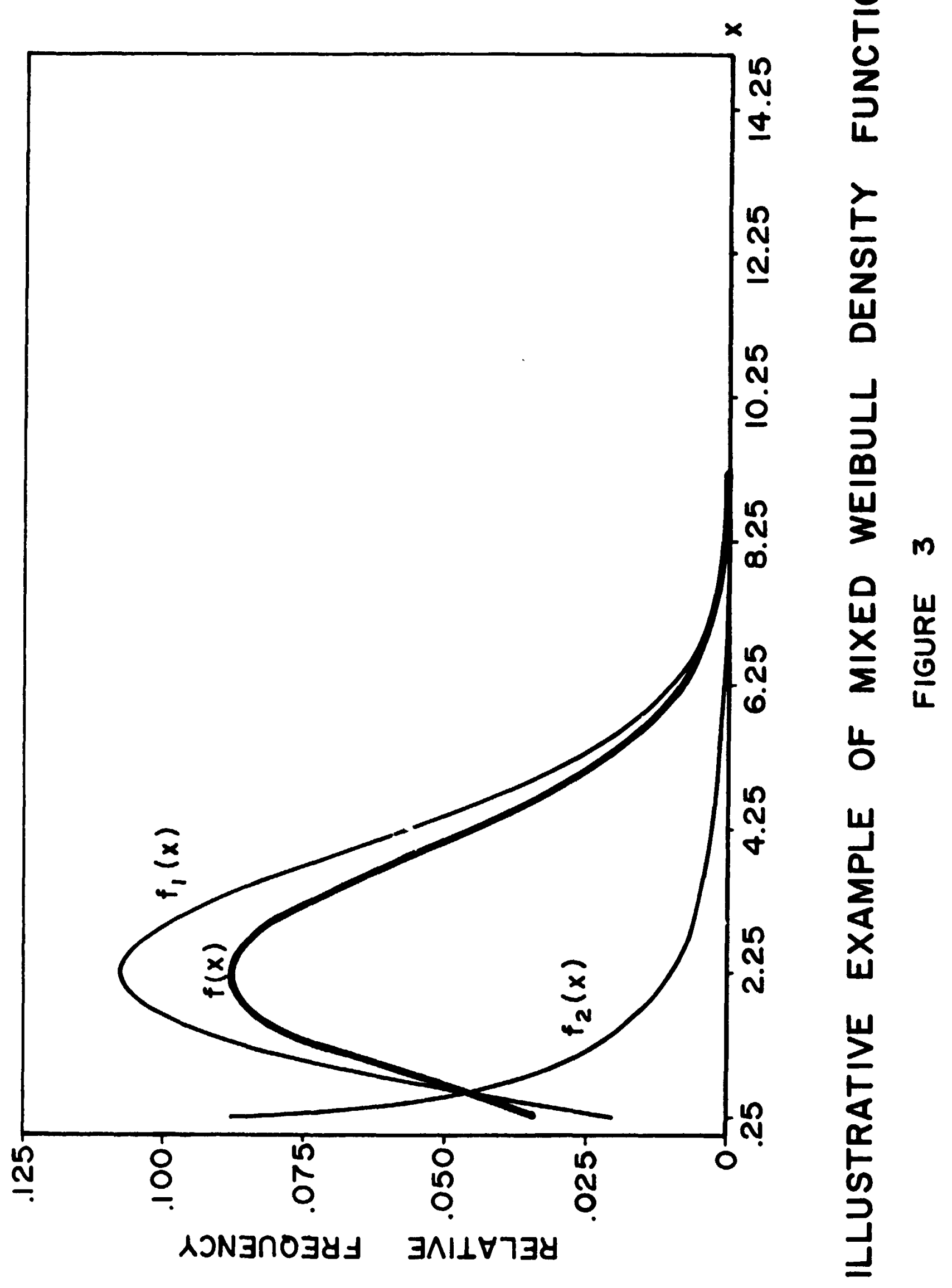




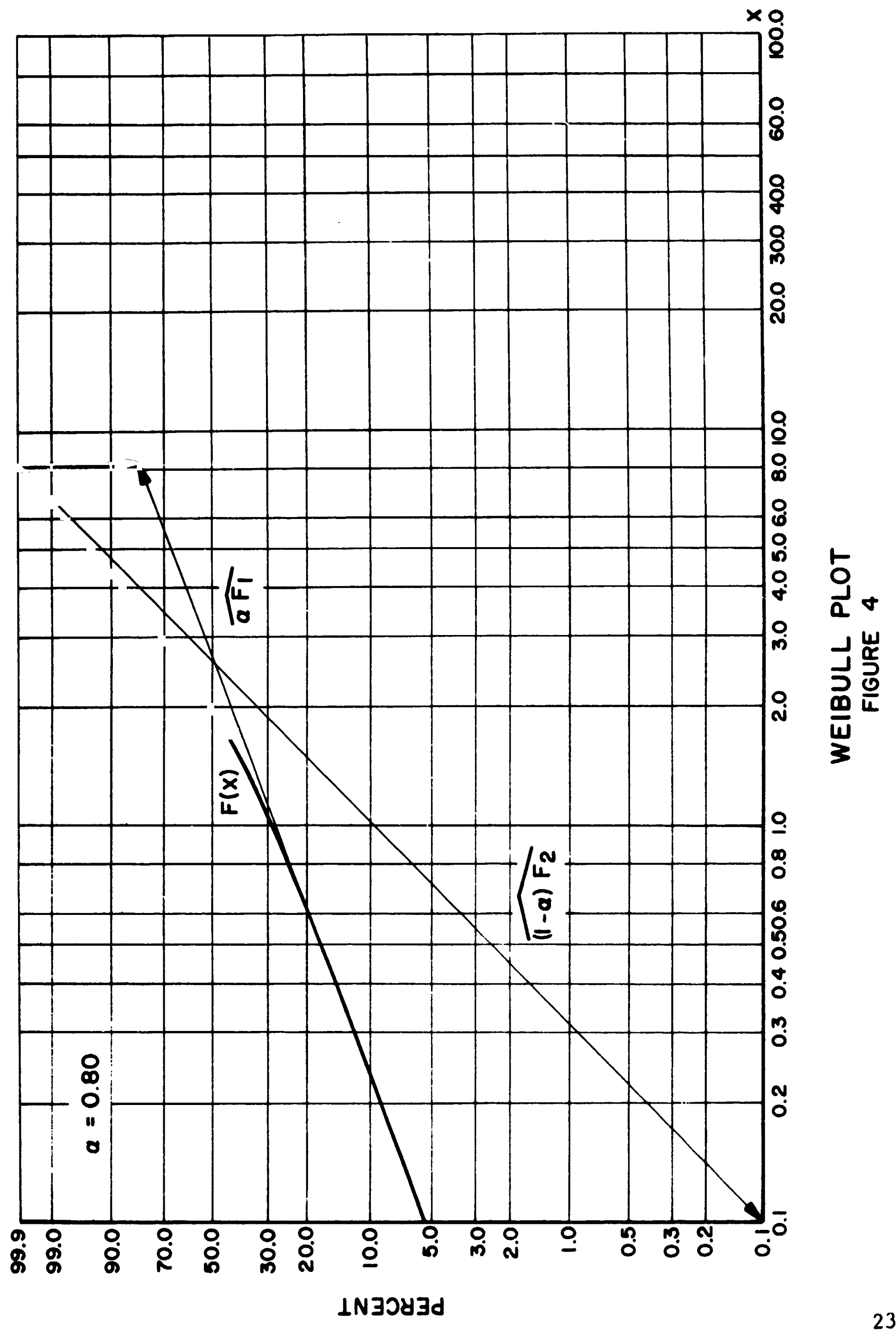


The computer program outlined in the Appendix gives all possible solutions to the estimating equations. Specifically, equations (12) and (13) produce four solutions resulting from the four combinations of the positive and negative signs prefixing the radicals; i.e., the combinations are $(-,-),(-,+),(+,-)$ and $(+,+)$. It was discovered that the computer program printout gave closest agreement between the theoretical moment $\mu_{5}$ and the sample moment $m_{5}^{\prime}$ when the combination $(-,-)$ was used.

Comparisons of expected with observed frequencies, along with a comparison of observed and expected distribution functions, are presented in Table II. It also seems appropriate to compare the observed frequencies for the mixed sample with these same frequencies assuming that the sample fits a simple Weibull distribution. This will prove or disprove that our mixed sample could be treated as a simple Weibull distribution. Cohen's maximum likelihood estimation procedure [4], was used to derive estimates for the parameters, and the resulting expected frequencies were obtained. Notations used in Table II are as follows:

$$
\begin{aligned}
\mathrm{f}_{\mathrm{o}}= & \text { observed frequencies for mixed data. } \\
\mathrm{F}_{\mathrm{O}}= & \text { observed distribution function for mixed data. } \\
\mathrm{f}_{\mathrm{e}}= & \begin{array}{l}
\text { expected frequencies using estimates derived in this } \\
\text { report. }
\end{array} \\
\mathrm{F}_{\mathrm{e}}= & \begin{array}{l}
\text { expected distribution function using estimates derived } \\
\text { in this report. }
\end{array} \\
\mathrm{f}_{\mathrm{es}}= & \text { expected frequencies assuming data fits a simple Weibull } \\
& \text { distribution. } \\
\mathrm{F}_{\mathrm{es}}= & \text { expected distribution function assuming data fits a simple } \\
& \text { Weibul1 distribution. }
\end{aligned}
$$

The agreement between observed frequencies for the sample and expected frequencies using the derived estimates is very good as shown in Table II. The corresponding observed and expected distribution functions are in very close agreement with the maximum absolute difference of 0.0050 occurring at the class $3.5-4.0$. Comparing this value with the Kolmogorov-Smirnov statistic, we see that

$$
D_{\alpha}^{N}=D_{.01}^{2000}=0.0364
$$

gives an excellent "goodness-of-fit" at the 99 percent level of confidence. 
TABLE II

OBSERVED AND EXPECTED FREQUENCIES FOR 2000 OBSERVATIONS FROM A MIXED WEIBULL DISTRIBU'TION

\begin{tabular}{|r|r|r|r|r|r|l|}
\hline \multicolumn{1}{c|}{ CLASSES } & \multicolumn{1}{c|}{$\mathrm{f}_{\mathrm{o}}$} & \multicolumn{1}{c|}{$\mathrm{f}_{\mathrm{e}}$} & \multicolumn{1}{c|}{$\mathrm{f}_{\mathrm{es}}$} & $\mathrm{F}_{\mathrm{o}}$ & $\mathrm{F}_{\mathrm{e}}$ & $\mathrm{F}_{\mathrm{es}}$ \\
\hline $0-0.5$ & 215 & 211.0 & 143.3 & .1075 & .1055 & .0717 \\
$0.5-1.0$ & 191 & 192.9 & 243.2 & .2030 & .2020 & .1932 \\
$1.0-1.5$ & 217 & 219.1 & 271.9 & .3115 & .3116 & .3292 \\
$1.5-2.0$ & 235 & 237.8 & 265.8 & .4290 & .4305 & .4621 \\
$2.0-2.5$ & 236 & 238.9 & 239.9 & .5470 & .5499 & .5821 \\
$2.5-3.0$ & 220 & 222.1 & 204.6 & .6570 & .6610 & .6843 \\
$3.0-3.5$ & 190 & 191.7 & 166.8 & .7520 & .7569 & .7677 \\
$3.5-4.0$ & 154 & 154.3 & 130.9 & .8290 & .8340 & .8332 \\
$4.0-4.5$ & 1.17 & 116.2 & 99.4 & .8875 & .8921 & .8828 \\
$4.5-5.0$ & 84 & 82.1 & 73.3 & .9295 & .9332 & .9195 \\
$5.0-5.5$ & 57 & 54.6 & 52.6 & .9580 & .9605 & .9458 \\
$5.5-6.0$ & 36 & 34.3 & 36.8 & .9760 & .9776 & .9642 \\
$6.0-6.5$ & 21 & 20.4 & 25.2 & .9865 & .9878 & .9768 \\
$6.5-7.0$ & 12 & 11.5 & 16.9 & .9925 & .9935 & .9852 \\
$7.0-7.5$ & 7 & 6.2 & 11.1 & .9960 & .9966 & .9908 \\
$7.5-8.0$ & 4 & 3.2 & 7.1 & .9980 & .9982 & .9943 \\
$8.0-8.5$ & 2 & 1.6 & 4.5 & .9990 & .9990 & .9966 \\
$8.5-9.0$ & 1 & .8 & 2.8 & .9995 & .9994 & .9980 \\
$9.0-9.5$ & 1 & .4 & 1.7 & 1.0000 & .9997 & .9988 \\
\hline
\end{tabular}

Comparing $F_{0}$ with $F_{e s}$ shows a maximum absolute difference in the distribution functions of 0.0358 occurring at the class $0-0.5$. Since the Kolmogorov-Smirnov statistic

$$
D_{\alpha}^{N}=D_{.05}^{2000}=0.0304
$$

this value of 0.0358 is sufficient to reject the hypothes is that our mixed distribution could be considered a simple Weibull distribution.

As an alternate goodness-of-fit test for agreement between observed frequencies and expected frequencies using the derived estimates, the $X^{\text {? }}$ index was calculated and the results are as follows: 


$$
X^{2}=1.2669 \quad \text { d.f. }=10 \quad P\left(X^{2}>1.2669=0.995\right) \text {. }
$$

Thus, in consideration of the low $x^{2}$ index of dispersion, we may conclude that we have an excellent fit for the chosen sample.

\section{CONCLUSIONS}

It is an accepted fact that the method of moments is not (except for distributions such as the normal, binomial, and Poisson) the most efficient procedure for estimating the parameters of a frequency distribution. Methods having maximum efficiency, such as the method of maximum likelihood are more desirable. However, in the case of the mixed Weibull distribution with its five parameters, the maximum likelihood estimating equations are almost intractable.

The central, noncentral, and factorial moments of this distribution were invest gated, and it was discovered that the noncentral moments possessed optimum characteristics for the development of estimating equations. A comparison of noncentral sample moments with the theoretical moments for the sample selected showed an error of 0.09 percent for the first moment, 0.44 percent for the second moment, 1.64 percent for the third moment, 4.94 percent for the fourth moment, and 12.73 percent for the fifth moment. This progressive increasing percentage of nonagreement between sample moments and theoretical moments illustrates the large sampling errors involved in the use of higher order moments. Using only the first three sample moments with their relatively low percentage of error in the estimating equations produced very good agreement between final estimates and the population parameters $\gamma_{1}, \gamma_{2}, \theta_{1}$ and $\theta_{2}$. Sheppard's corrections for grouped data were applied to the sample moments in order to increase this agreement, but produced no significant change in the results; therefore, the corrections were not used in the estimating equations.

This paper presents an estimating procedure that produced very good results for the sample chosen. The use of electronic digital computers makes the somewhat involved method practical and applicable to experiments in which the mixed Weibull distribution is the appropriate statisticai model.

In the author's opinion, the estimating prosedures outlined in this report warrant further investigation for increase in efficiency, and improvement and simplification of form for the estimating equations involved. 


\section{APPENDIX \\ COMPUTER PROGRAM FOR ESTIMATING THE PARAMETERS \\ OF A MIXED WEIBULL DISTRIBUTION}

The author wishes to acknowledge the assistance of Mr. Raymond

Smith of the Computation Laboratory, MSFC, for his work on the CDC 3200 computer program. 


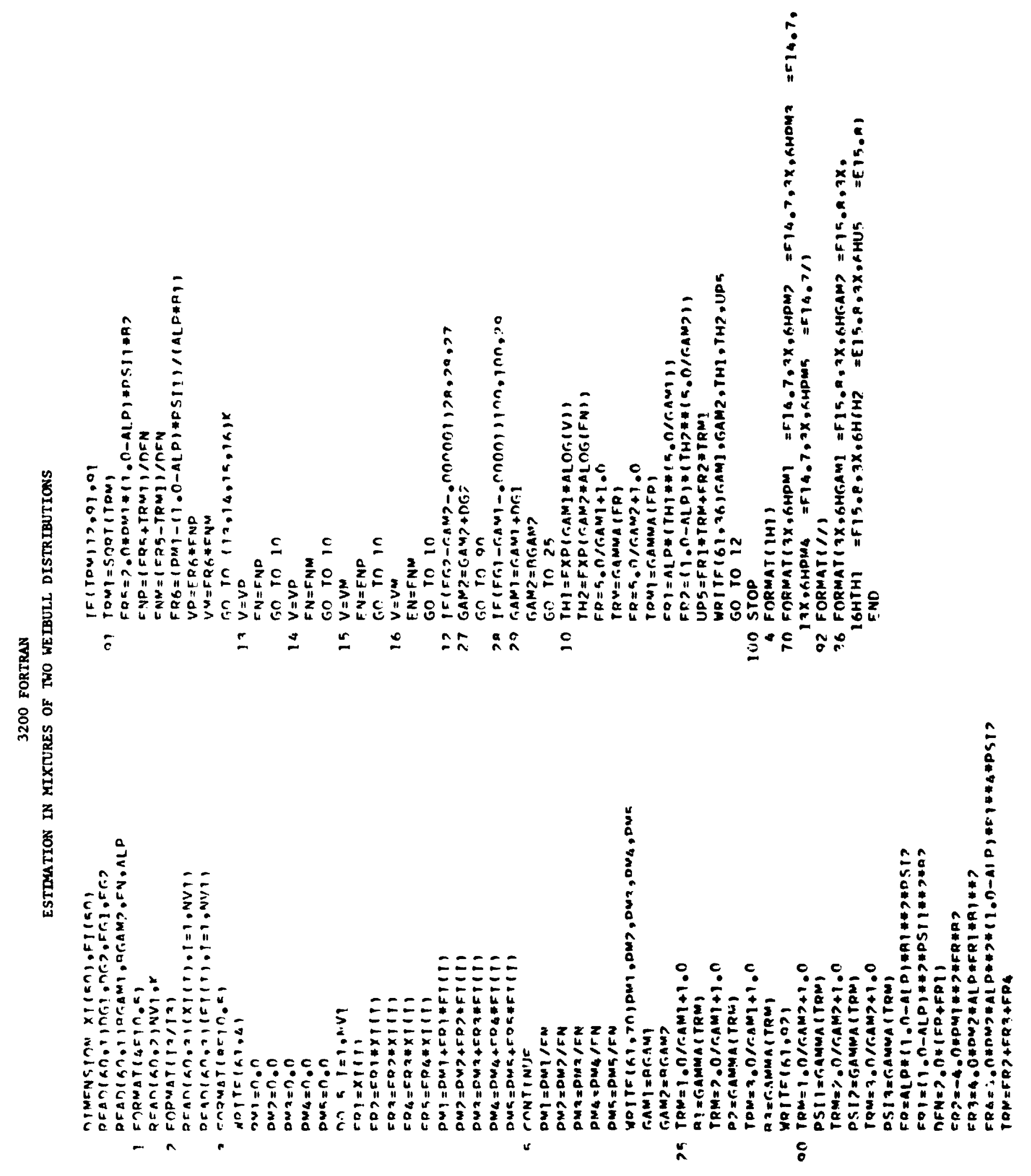



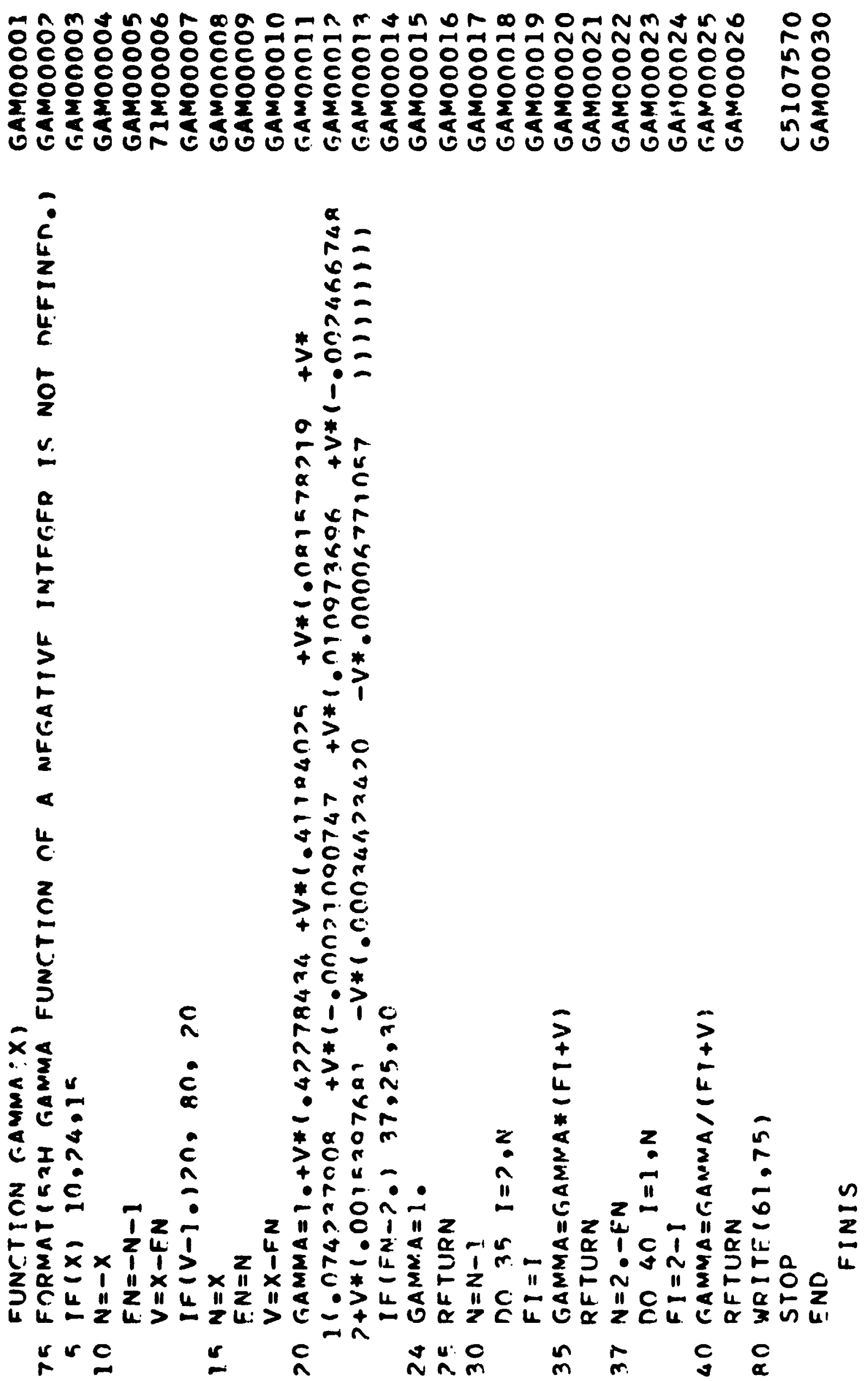


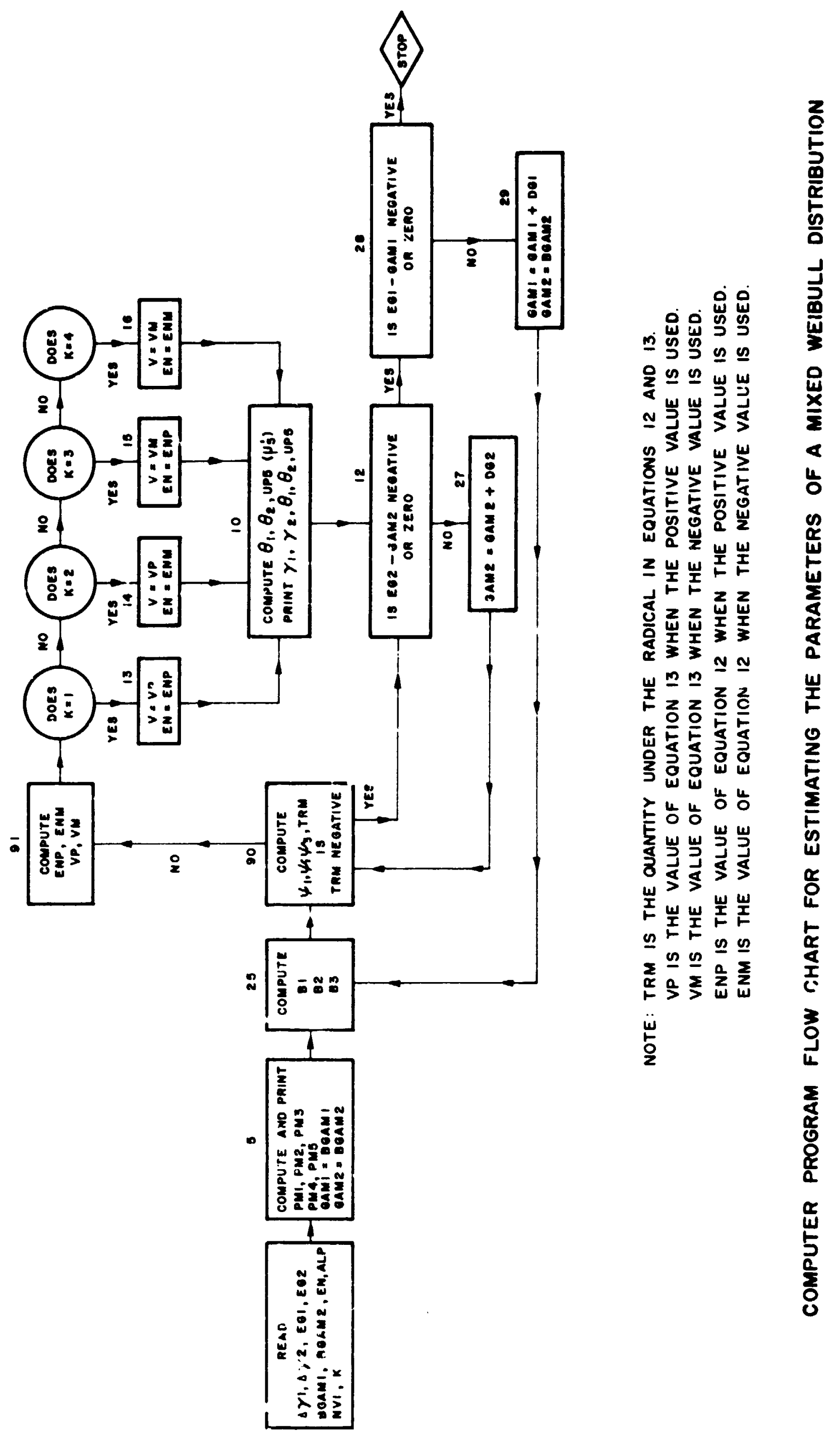


LOAD DATA

FIRST, TWO TABLES ARE READ IN STARTING IN XI AND FI

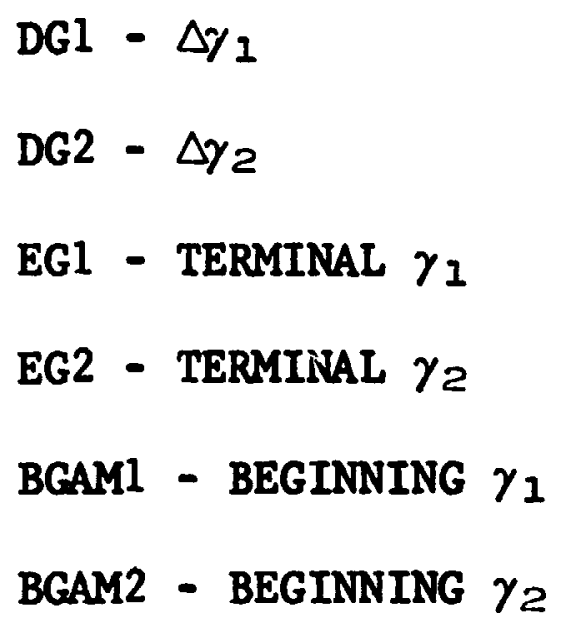




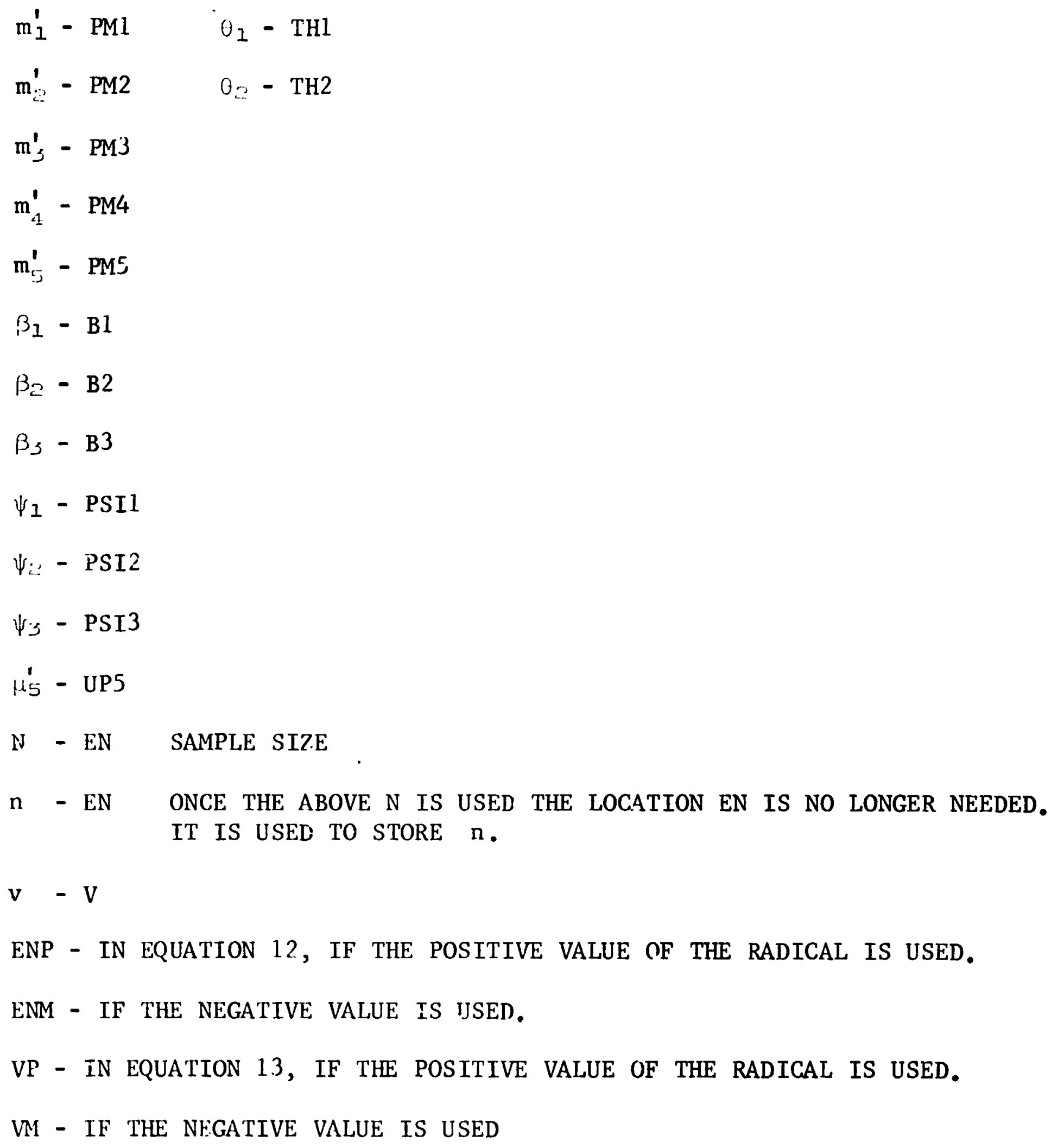

THE FOLLOWING FOUR COMBINATIONS ARE POSSIBLE, DEPENDING UPON WHAT NUMBER IS READ INTO LOCATION K:

$v \quad n$ REGARDLESS OF WHICH COMBINATION IS USED, BEFORE THE ++1 PROGRAM IS CONTINUED, THE VALUE OF EQUATION 13 IS

$+\quad 2$ STORED IN V AND THE VATUE OF EQUATION 12 IS STORED

-+3 IN EN. 


\section{REFERENCES}

1. Cohen, A. Clifford, Jr., "Estimati, I in Mixtures of Two Normal Distributions," University of Georgia, Institute of Statistics, TR No. 13, 1965.

2. Cohen, A. Clifford, Jr., "Estimation in Mixtures of Poisson and Mixtures of Exponential Distributions," NASA Technical Memorandum, IM X-53245, April 9, 1965, Unclassified.

3. Cohen, A. Clifford, Jr., "Estimation in a Mixture of a Poisson with a Negative Binc al Distribution," University of Georgia, Institute of Statistics, … Nu. 15, 1965.

4. Cohen, A. Clifford, Jr., "Maxinum Likelihood Estimation in the Weibull Distribution Based on Complete and on Censored Samples," University of Georgia, Institute of Statistics.

5. Hoel, Paul G., Introduction to Mathematical Statistics, John Wiley and Sons, New York, 1963.

6. Kao, J. H. K., "A Graphical Estimation of Mixed Weibull Parameters in Life-Testing of Electron Tubes," Technometrics, Vol. I, 1959, pp. 389-407.

7. Rider, Paul R., "Estimating the Parameters of Mixed Poisson, Binomial and Weibull Distributions by the Method of Moments," Bulletin de l'institute international de statistique, 38, part 2.

8. Whittaker and Robinson, The Calculus of Observations, Blackie and Son, Ltd., London.

9. Whittaker and Watson, Modern Analysis, Cambridge University Press, Cambridge, 1958.

10. Menon, M. V., "Estimation of the Shape and Scale Parameters of the Weibull Distribution," Technometrics, Vol. 5, No. 2, 1963, pp. 175182.

11. Kao, J. H. K., "Computer Methods for Estimating Weibull Parameters in Reliability Studies," Institute of Radio Engineers Transactions on Reliability and Quality Control, Pp. 15-22, PGRQC-13, July 1958. 


\section{REFERENCES (Continued)}

12. Kenda11, M. G. and A. Stuart, 1958. The Advanced Theory of Statistics, Vol. 1, Ha fner Publishing Co., New York, N. Y.

13. Kenda11, M. G. and A. Stuart, 1958. The Advanced Theory of Statistics, Vol. 2, Hafner Publishing Co., New York, N. Y.

14. Kenney, J. F. and E. S. Keeping, 1939. Mathematics of Statistics, Part 2, D. Van Nostrand Co., Inc., Princeton, New Jersey.

15. Pearson, Kar1, "Contributions to the Mathematical Theory of Evolution," Philosophical Transactions of the Royal Society, London, Vol. 185, 1894 pp. 71-110. 


\section{ESTIMATION OF PARAMETERS IN COMPOUND WEIBULL DISTRIBUTIONS}

by Lee W. Falls

The information in this report has been reviewed for security classification. Review of any information concerning Department of Defense or Atomic Energy Commission programs has been made by the MSFC Security Classification officer. This report, in its entirety, has been determined to be unclassified.

This document has also been reviewed and approved for technical accuracy.

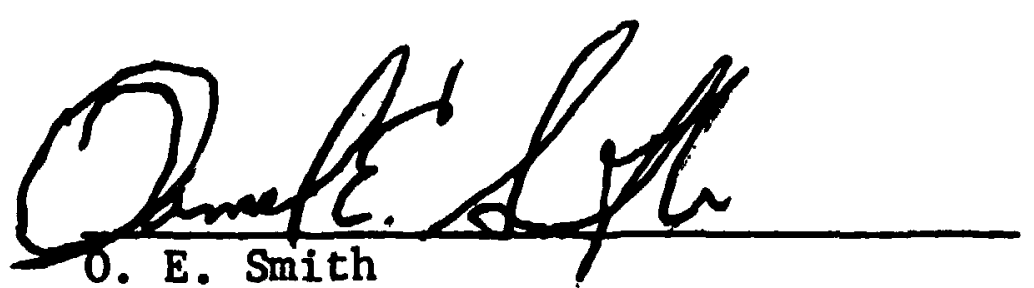

Chief, Terrestrial Environment Branch

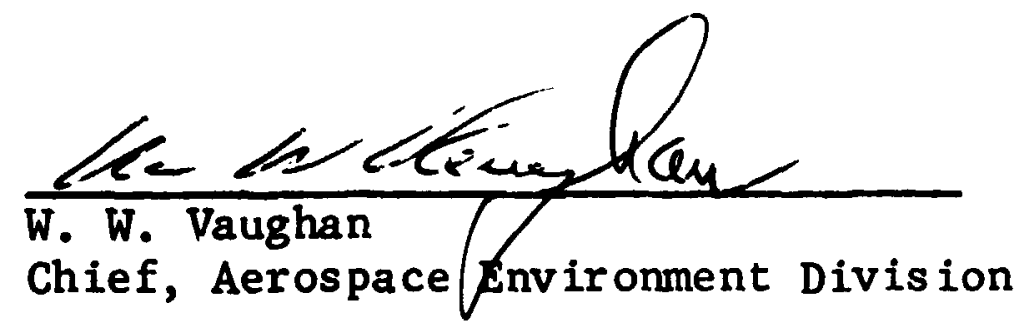

\footnotetext{
Cousach

E. D. Geissler

Director, Aero-As trodynamics Laboratory
} 